\title{
On the Dimension of the Singular Set of Solutions to the Navier-Stokes Equations
}

\author{
James C. Robinson, Witold Sadowski ${ }^{\star}$ \\ Mathematics Institute, University of Warwick, Coventry CV4 7AL, UK. \\ E-mail: Sadowski@warwick.ac.uk \\ J. C. Robinson \\ E-mail: Robinson@warwick.ac.uk
}

Received: 23 December 2010 / Accepted: 10 April 2011

Published online: 24 September 2011 - (C The Author(s) 2011. This article is published with open access

at Springerlink.com

\begin{abstract}
In this paper we prove that if a suitable weak solution $u$ of the NavierStokes equations is an element of $L^{w}\left(0, T ; L^{s}\left(\mathbb{R}^{3}\right)\right)$, where $1 \leq 2 / w+3 / s \leq 3 / 2$ and $3<w, s<\infty$, then the box-counting dimension of the set of space-time singularities is no greater than $\max \{w, s\}(2 / w+3 / s-1)$. We also show that if $\nabla u \in L^{w}\left(0, T ; L^{s}(\Omega)\right)$ with $2<s \leq w<\infty$, then the Hausdorff dimension of the singular set is bounded by $w(2 / w+3 / s-2)$. In this way we link continuously the bounds on the dimension of the singular set that follow from the partial regularity theory of Caffarelli, Kohn, \& Nirenberg (Commun. Pure Appl. Math. 35:771-831, 1982) to the regularity conditions of Serrin (Arch. Ration. Mech. Anal. 9:187-191, 1962) and Beirão da Veiga (Chin. Ann. Math. Ser. B 16(4):407-412, 1995).
\end{abstract}

\section{Introduction}

The flow of an incompressible fluid in a domain $\Omega \subseteq \mathbb{R}^{3}$ is governed by the system of the Navier-Stokes equations:

$$
\begin{gathered}
u_{t}-\Delta u+(u \cdot \nabla) u+\nabla p=0, \quad \operatorname{div} u=0, \\
u(0)=u_{0},\left.\quad u\right|_{\partial \Omega}=0,
\end{gathered}
$$

where $u$ is the velocity of the fluid and $p$ is the pressure. Since the works of Leray [10], Hopf [7] and Ladyzhenskaya [9], it is known that each divergence-free initial condition $u_{0} \in \mathbb{L}^{2}(\Omega):=\left[L^{2}(\Omega)\right]^{3}$ gives rise to a weak solution $u \in L^{\infty}\left(0, T ; \mathbb{L}^{2}\right) \cap L^{2}\left(0, T ; \mathbb{H}_{0}^{1}\right)$ that satisfies the Navier-Stokes equations in the distributional sense. The open question is whether or not each initial condition $u_{0} \in \mathbb{H}_{0}^{1}$ gives rise to a weak solution that is actually strong: $u \in L^{\infty}\left(0, T ; \mathbb{H}_{0}^{1}\right) \cap L^{2}\left(0, T ; \mathbb{H}^{2}\right)$.

^ Permanenet address: Faculty of Mathematics, Informatics and Mechanics, University of Warsaw, Banacha 2 02-097, Warszawa, Poland. 
There are many results that give sufficient conditions for regularity of a weak solution $u$. The simplest is due to Serrin [17] and says that $u$ is regular if $u \in L^{w}\left(0, T ; L^{s}(\Omega)\right)$ with

$$
\frac{2}{w}+\frac{3}{s} \leq 1
$$

(In fact Serrin's proof requires strict inequality in (3); that the condition in (3) is sufficient was shown by Fabes, Jones, \& Rivière [5] with the exception of the endpoint cases; Struwe [18] gave an alternative proof including $u \in L^{2}\left(0, T ; L^{\infty}\right)$; and Escauriaza, Seregin, \& Šverák showed relatively recently in [4] that $u \in L^{\infty}\left(0, T ; L^{3}\right)$ implies regularity).

Notice that the Sobolev embedding result $H^{1}(\Omega) \subset L^{6}(\Omega)$ implies that any weak solution is an element of $L^{2}\left(0, T ; L^{6}\right)$ which gives $2 / w+3 / s=3 / 2$. Even though this 'regularity gap' of $1 / 2$ prevents one from proving the regularity of weak solutions, it has been shown by many authors that the putative set $\mathcal{S}$ of points at which $u$ is not regular must be very small. For example, it is known that for a suitable weak solution the set of singular points in space-time has one-dimensional parabolic Hausdorff measure zero (Caffarelli, Kohn, \& Nirenberg, [2]) and box-counting dimension no greater than 5/3 (Robinson \& Sadowski [15]; see also Kukavica [8], for a finer result).

In this paper we present a link between these partial regularity results bounding the dimension of the set of space-time singularities, and the regularity result of Serrin (et al.). More precisely, in our first result we consider a suitable weak solution $u$ with $u \in L^{w}\left(0, T ; L^{s}\left(\mathbb{R}^{3}\right)\right)$ where

$$
1 \leq \frac{2}{w}+\frac{3}{s} \leq \frac{3}{2}
$$

and $3<w, s<\infty$; as a consequence of the results of Caffarelli et al. we prove that the box-counting dimension of the set of space-time singularities is no greater than

$$
\alpha=\max \{w, s\}\left(\frac{2}{w}+\frac{3}{s}-1\right) .
$$

Specialising this to the case $w=s$ shows that if $u \in L^{s}\left((0, T) \times \mathbb{R}^{3}\right)$, then $d_{B}(\mathcal{S}) \leq 5-s$. Observing that any weak solution belongs to $L^{10 / 3}\left((0, T) \times \mathbb{R}^{3}\right)$ since

$$
\int_{0}^{T}\|u(t)\|_{L^{10 / 3}}^{10 / 3} \mathrm{~d} t \leq \int_{0}^{T}\|u(t)\|_{L^{2}}^{4 / 3}\|u(t)\|_{L^{6}}^{2} \mathrm{~d} t
$$

(by Hölder's inequality), we recover the bound $d_{B}(\mathcal{S}) \leq 5 / 3$ for weak solutions, which decreases as $s$ increases until we reach the critical value $s=5$ known to guarantee regularity from (3).

While this result requires us to consider the equations on $\mathbb{R}^{3}$ due to problems estimating the pressure, we can circumvent this and consider the equations on a bounded domain if we instead impose conditions on $\nabla u$. In our second result we consider a suitable weak solution $u$ such that

$$
\nabla u \in L^{w}\left(0, T ; L^{s}(\Omega)\right) \quad \text { with } 2 \leq \frac{2}{w}+\frac{3}{s} \leq \frac{5}{2} .
$$

We prove that if $2<s \leq w<\infty$, then the Hausdorff dimension of the singular set of $u$ is no greater than

$$
\beta=w\left(\frac{2}{w}+\frac{3}{s}-2\right) .
$$


This result provides a link between the partial regularity result in its standard form (a bound on the Hausdorff dimension of the singular set) and the condition for regularity due to Beirão da Veiga [1], that $u$ is regular if $2 / w+3 / s=2$. Again, in the case of weak solutions we have $w=s=2$ which yields the partial regularity result in its standard form (the Hausdorff dimension of the singular set is no larger than one), and we recover regularity when $w=s=5 / 2$.

\section{Notation and Auxiliary Results}

Throughout the paper we use standard notation for Lebesgue and Sobolev spaces. The Bochner space $L^{w}\left(0, T ; L^{s}(\Omega)\right)$ is endowed with the norm:

$$
\|u\|_{L^{w}\left(0, T ; L^{s}(\Omega)\right)}=\int_{0}^{T}\|u(t)\|_{L^{s}(\Omega)}^{w} \mathrm{~d} t .
$$

We denote by $Q_{r}(x, t)$ the space-time cylinder

$$
Q_{r}(x, t)=B_{r}(x) \times\left(t-r^{2}, t\right),
$$

where $B_{r}(x)$ is a three-dimensional ball of radius $r>0$ centred at $x$.

In what follows we consider only suitable weak solutions, which are weak solutions that in addition satisfy a local energy inequality and for which the associated pressure belongs to $L^{5 / 3}\left(Q_{T}\right)$, where $Q_{T}$ is the space-time domain.

We say that $z \in \mathbb{R}^{3} \times \mathbb{R}_{+}$is a regular point of a suitable weak solution $u$ if $u$ is bounded in some neighbourhood of $z$. A point is singular if it is not regular, and the set of all singular points of a suitable weak solution $u$ we denote by $\mathcal{S}$.

For our main results we will need the following two lemmas. The first formalises the fact that in some sense $p \sim u^{2}$. We do not know how to prove a similar result on a bounded domain; this is the reason that we restrict to the whole of $\mathbb{R}^{3}$ in Theorem 1 . However, a recent result due to Wolf [19] shows that one can remove the pressure term from the condition of regularity used in the proof of Lemma 2 allowing its generalisation to bounded domains.

Lemma 1. If $u \in L^{w}\left(0, T ; L^{s}\left(\mathbb{R}^{3}\right)\right)$, then $p \in L^{w / 2}\left(0, T ; L^{s / 2}\left(\mathbb{R}^{3}\right)\right)$.

Proof. Equations (1) and (2) imply formally that

$$
\Delta p=-\sum_{i, j} \frac{\partial^{2}}{\partial x_{i} \partial x_{j}}\left(u^{i} u^{j}\right)
$$

From the Calderon-Zygmund theorem we can now deduce that for all $2<s<\infty$,

$$
\int_{\mathbb{R}^{3}}|p|^{s / 2} \mathrm{~d} x \leq C(s) \int_{\mathbb{R}^{3}}|u|^{s} \mathrm{~d} x,
$$

and therefore

$$
\int_{0}^{T}\|p(t)\|_{L^{s / 2}}^{w / 2} \mathrm{~d} t \leq \int_{0}^{T}\|u(t)\|_{L^{s}}^{w} \mathrm{~d} t
$$

(see Caffarelli et al., 1982, for details). 
The second simple lemma - essentially a version of the regularity criterion of Caffarelli et al. obtained by repeated application of Hölder's inequality - is the key observation that allows us to prove our main results.

Lemma 2. Assume that $u$ is a suitable weak solution to the Navier-Stokes equations and $u \in L^{w}\left(0, T ; L^{s}\left(\mathbb{R}^{3}\right)\right)$ with $3 \leq w, s<\infty$. There exists an absolute constant $\varepsilon>0$ such that if $z=(x, t)$ is a singular point of $u$ then

$$
\varepsilon r^{w\left(\frac{3}{s}+\frac{2}{w}-1\right)} \leq \int_{t-r^{2}}^{t}\left(\int_{B_{r}(x)}|u|^{s} \mathrm{~d} x\right)^{w / s} \mathrm{~d} t+\int_{t-r^{2}}^{t}\left(\int_{B_{r}(x)}|p|^{s / 2} \mathrm{~d} x\right)^{w / s} \mathrm{~d} t
$$

for all sufficiently small $r>0$.

Proof. The fundamental regularity result of Caffarelli et al. [2] is that there exists an absolute constant $\epsilon_{0}$ such that if for any $r>0$ such that $Q_{r}(x, t) \subset \mathbb{R}_{+} \times \mathbb{R}^{3}$,

$$
\frac{1}{r^{2}} \int_{Q_{r}(x, t)}|u|^{3} \mathrm{~d} x \mathrm{~d} t+\frac{1}{r^{2}} \int_{Q_{r}(x, t)}|p|^{3 / 2} \mathrm{~d} x \mathrm{~d} t<2 \epsilon_{0},
$$

then $z=(x, t)$ is regular. It follows that if $z$ is a singular point, then for all sufficiently small $r>0$ we have

$$
\epsilon_{0} \leq \frac{1}{r^{2}} \int_{Q_{r}(x, t)}|u|^{3} \mathrm{~d} x \mathrm{~d} t \quad \text { or } \quad \epsilon_{0} \leq \frac{1}{r^{2}} \int_{Q_{r}(x, t)}|p|^{3 / 2} \mathrm{~d} x \mathrm{~d} t .
$$

From Hölder's inequality it follows that for all $s \geq 3$ :

$$
\begin{aligned}
r^{-2} \int_{Q_{r}(x, t)}|u|^{3} \mathrm{~d} x \mathrm{~d} t & \leq r^{-2} \int_{t-r^{2}}^{t}\left(\int_{B_{r}(x)}|u|^{s} \mathrm{~d} x\right)^{\frac{3}{s}}\left(\int_{B_{r}(x)} \mathrm{d} x\right)^{1-\frac{3}{s}} \mathrm{~d} t \\
& \leq c r^{1-\frac{9}{s}}\left[\int_{t-r^{2}}^{t}\left(\int_{B_{r}(x)}|u|^{s} \mathrm{~d} x\right)^{w / s} \mathrm{~d} t\right]^{3 / w}\left[\int_{t-r^{2}}^{t} \mathrm{~d} t\right]^{1-\frac{3}{w}} .
\end{aligned}
$$

Hence

$$
\frac{1}{r^{2}} \int_{Q_{r}(x, t)}|u|^{3} \mathrm{~d} x \mathrm{~d} t \leq c r^{3-\frac{9}{s}-\frac{6}{w}}\left[\int_{t-r^{2}}^{t}\left(\int_{B_{r}(x)}|u|^{s} \mathrm{~d} x\right)^{w / s} \mathrm{~d} t\right]^{3 / w} .
$$

Similarly, for $w \geq 3$, we obtain:

$$
\frac{1}{r^{2}} \int_{Q_{r}(x, t)}|p|^{3 / 2} \mathrm{~d} x \mathrm{~d} t \leq c r^{3-\frac{9}{s}-\frac{6}{w}}\left[\int_{t-r^{2}}^{t}\left(\int_{B_{r}(x)}|p|^{s / 2} \mathrm{~d} x\right)^{w / s} \mathrm{~d} t\right]^{3 / w} .
$$

Therefore taking $\varepsilon=\epsilon_{0}^{w / 3} c^{-w / 3}$ we obtain the assertion of the lemma.

Notice that from Lemma 2 it follows immediately that if $u \in L^{w}\left(0, T ; L^{s}\right)$ with $3 / w+2 / s=1$ and $w, s>3$ then it is regular. Indeed, it follows from (3),(4) and (5) that if $2 / w+3 / s=1$ then there exits a sequence $r_{n} \rightarrow 0$ such that for each $n$ we have

$$
\begin{aligned}
\varepsilon & \leq \int_{t-r_{n}^{2}}^{t}\left(\int_{B_{r_{n}}(x)}|u|^{s} \mathrm{~d} x\right)^{w / s} \mathrm{~d} t+\int_{t-r_{n}^{2}}^{t}\left(\int_{B_{r_{n}}(x)}|p|^{s / 2} \mathrm{~d} x\right)^{w / s} \mathrm{~d} t \\
& \leq \int_{t-r_{n}^{2}}^{t}\|u\|_{L^{s}\left(\mathbb{R}^{3}\right)}^{w}+\|p\|_{L^{s / 2}\left(\mathbb{R}^{3}\right)}^{w / 2} \mathrm{~d} t,
\end{aligned}
$$

which contradicts the fact that $u \in L^{w}\left(0, T ; L^{s}\left(\mathbb{R}^{3}\right)\right)$ and $p \in L^{w / 2}\left(0, T ; L^{s / 2}\left(\mathbb{R}^{3}\right)\right)$. 


\section{Main Results}

In this section we give our main results concerning the dimension of the singular set. The classical result of Caffarelli, Kohn, \& Nirenberg is given in terms of the parabolic Hausdorff measure. For a given $s \geq 0$, let

$$
\mathcal{P}_{\delta}^{s}(X)=\inf \left\{\sum_{j} r_{j}^{s}: X \subset \cup_{j=1}^{\infty} Q_{r_{j}}\left(x_{j}, t_{j}\right), r_{j}<\delta\right\},
$$

and define the $s$-dimensional parabolic Hausdorff measure as

$$
\mathcal{P}^{s}(X)=\lim _{\delta \rightarrow 0} \mathcal{P}_{\delta}^{s}(X) .
$$

One can define a parabolic Hausdorff dimension $d_{P H}(X)=\inf \left\{s: \mathcal{P}^{s}(X)=0\right\}$; if $\mathcal{P}^{s}(X)<\infty$ then $d_{P H}(X) \leq s$. This quantity also bounds the standard Hausdorff dimension $d_{H}$ (which can be defined in the same way but with cylinders replaced by balls), $d_{H}(X) \leq d_{P H}(X)$.

We will also make use of the upper box-counting dimension $d_{B}(X)$. Let $N(X, \epsilon)$ denote the minimum number of balls of radius $\epsilon$ necessary to cover $X$; then

$$
d_{B}(X)=\limsup _{\epsilon \rightarrow 0} \frac{\log N(X, \epsilon)}{-\log \epsilon} .
$$

A useful observation here is that one obtains the same quantity if $N(X, \epsilon)$ instead denotes the maximum number of disjoint balls of radius $\epsilon$ with centres in $X$. One always has $d_{H}(X) \leq d_{B}(X)$. See Falconer [6] or Robinson [13] for details.

Theorem 1. Assume that $u$ is a suitable weak solution with $u \in L^{w}\left(0, T ; L^{s}\left(\mathbb{R}^{3}\right)\right)$ for $3<w, s<\infty$. Then the box-counting dimension of its singular set $\mathcal{S}$ is no greater than

$$
\alpha=\max \{w, s\}\left(\frac{2}{w}+\frac{3}{s}-1\right) .
$$

Proof. For a given sufficiently small $r>0$ let $N(r)$ be the maximal number of disjoint 4-dimensional balls of radius $2 r$ centred at points $z_{i}=\left(x_{i}, t_{i}\right) \in \mathcal{S}$, where $i=$ $1,2,3, \ldots, N(r)$. Observe that for all sufficiently small $r$ the cylinders $Q_{r}\left(x_{i}, t_{i}\right)$ are disjoint, too.

For $i=1,2, \ldots, N(r)$ we define the function $a_{i}$ by

$$
a_{i}(t)=\int_{B_{r}\left(x_{i}\right)}|u(t, x)|^{s} \mathrm{~d} x \quad \text { if } t_{i}-r^{2} \leq t \leq t_{i},
$$

and we let $a_{i}(t)=0$ for all other values of $t$. Similarly, for $i=1,2, \ldots, N(r)$ we define functions $b_{i}$ by

$$
b_{i}(t)=\int_{B_{r}\left(x_{i}\right)}|p(t, x)|^{s / 2} \mathrm{~d} x \quad \text { if } \quad t_{i}-r^{2} \leq t \leq t_{i},
$$

and $b_{i}(t)=0$ otherwise.

Notice that for each $i=1,2, \ldots, N(r)$ we have

$$
\int_{t_{i}-r^{2}}^{t_{i}}\left(\int_{B_{r}\left(x_{i}\right)}|u|^{s} \mathrm{~d} x\right)^{w / s} \mathrm{~d} t=\int_{0}^{T}\left[a_{i}(t)\right]^{w / s} \mathrm{~d} t
$$


and

$$
\int_{t_{i}-r^{2}}^{t_{i}}\left(\int_{B_{r}\left(x_{i}\right)}|p|^{s / 2} \mathrm{~d} x\right)^{w / s} \mathrm{~d} t=\int_{0}^{T}\left[b_{i}(t)\right]^{w / s} \mathrm{~d} t .
$$

Since the cylinders are disjoint we have

$$
\int_{0}^{T}\left(\sum_{i=1}^{N(r)} a_{i}(t)\right)^{w / s}+\left(\sum_{i=1}^{N(r)} b_{i}(t)\right)^{w / s} \mathrm{~d} t \leq M,
$$

where

$$
M=\int_{0}^{T}\left(\int_{\mathbb{R}^{3}}|u|^{s} \mathrm{~d} x\right)^{w / s} \mathrm{~d} t+\int_{0}^{T}\left(\int_{\mathbb{R}^{3}}|p|^{s / 2} \mathrm{~d} x\right)^{w / s} \mathrm{~d} t .
$$

Now we are going to consider the cases $w \geq s$ and $s>w$ separately.

Case 1. If $w \geq s$ then (3) implies that

$$
\sum_{i=1}^{N(r)} \int_{0}^{T}\left[a_{i}(t)\right]^{w / s}+\left[b_{i}(t)\right]^{w / s} \mathrm{~d} t \leq M .
$$

From Lemma 2 it now follows that

$$
N(r) \varepsilon r^{w\left(\frac{2}{w}+\frac{3}{s}-1\right)} \leq M .
$$

If the box-counting dimension of $\mathcal{S}$ was greater than $\alpha=w\left(\frac{2}{w}+\frac{3}{s}-1\right)$, then for some constant $\delta>0$ there would exist a sequence $r_{n} \rightarrow 0$ such that $N\left(\mathcal{S}, r_{n}\right)>r_{n}^{-\alpha-\delta}$. For $n \rightarrow \infty$ the left-hand side of (8) would tend to infinity, giving a contradiction.

Case 2. If $w<s$ then from Hölder's inequality it follows that

$$
N^{w / s-1}\left(\sum_{i=1}^{N}\left[a_{i}\right]^{w / s}+\left[b_{i}\right]^{w / s}\right) \leq\left(\sum_{i=1}^{N} a_{i}\right)^{w / s}+\left(\sum_{i=1}^{N} b_{i}\right)^{w / s} .
$$

Thus we have

$$
N(r) N(r)^{r / s-1} \varepsilon r^{w\left(\frac{2}{w}+\frac{3}{s}-1\right)} \leq M,
$$

and the proof is concluded as before.

As remarked in the Introduction, every suitable weak solution belongs to $L^{10 / 3}\left((0, T) \times \mathbb{R}^{3}\right)$, and so has a singular set whose box-counting dimension is no greater than 5/3 (cf. Robinson \& Sadowski [15]). At the other extreme, if $u$ satisfies Serrin's condition (e.g. $u \in L^{5}\left((0, T) \times \mathbb{R}^{3}\right)$ then the singular set has box-counting dimension zero. (In fact we showed after the proof of Lemma 2 that in this case the singular set is empty.)

We now provide a related result which, inspired by Beirão da Veiga [1], makes an assumption on $\nabla u$ rather than on $u$ itself. The advantage of this is that the following theorem is also valid on bounded domains, since the regularity condition involved does not require any properties of the pressure. 
Theorem 2. Assume that $u$ is a suitable weak solution with

$$
\nabla u \in L^{w}\left(0, T ; L^{s}(\Omega)\right),
$$

where $2<s \leq w<\infty$. Then

$$
\mathcal{P}^{\beta}(\mathcal{S})=0
$$

where

$$
\beta=w\left(\frac{2}{w}+\frac{3}{s}-2\right) .
$$

Proof. For this result we use Caffarelli et al.'s second regularity theorem (which is what allows them to deduce that $\mathcal{P}^{1}(\mathcal{S})=0$ for any weak solution), namely that there exists an absolute constant $\varepsilon>0$ such that if

$$
\limsup _{r \rightarrow 0} \frac{1}{r} \int_{Q_{r}(x, t)}|\nabla u|^{2} \mathrm{~d} x \mathrm{~d} t \leq \varepsilon
$$

then $(x, t)$ is regular.

Fix $\delta>0$, and for each singular point $z=(x, t) \in \mathcal{S}$ choose a cylinder $Q_{r}(x, t)$ with $r<\delta / 5$ such that

$$
\frac{1}{r} \int_{Q_{r}(x, t)}|\nabla u|^{2} \mathrm{~d} x \mathrm{~d} t \geq \varepsilon
$$

let $\mathcal{C}$ be the family of all these cylinders. Using the covering lemma from Caffarelli et al. we can choose a countable subfamily $\mathcal{C}^{\prime}$ of disjoint cylinders $Q_{r_{i}}\left(z_{i}\right)$ such that $Q_{5 r_{i}}\left(z_{i}\right)$ still covers the singular set $\mathcal{S}$.

To show that $d_{P H}(\mathcal{S}) \leq \beta$, it is sufficient to show that $\mathcal{P}^{\beta}(\mathcal{S})<\infty$. To this end we notice that

$$
\begin{aligned}
\int_{Q_{r_{i}}\left(x_{i}, t_{i}\right)}|\nabla u|^{2} \mathrm{~d} x \mathrm{~d} t & \leq \int_{t_{i}-r_{i}^{2}}^{t_{i}}\left(\int_{B_{r_{i}}\left(x_{i}\right)}|\nabla u|^{s} \mathrm{~d} x\right)^{2 / s}\left(\int_{B_{r_{i}}\left(x_{i}\right)} \mathrm{d} x\right)^{1-\frac{2}{s}} \mathrm{~d} t \\
& \leq c\left(r_{i}\right)^{3-\frac{6}{s}}\left(\int_{t_{i}-r_{i}^{2}}^{t_{i}}\left(\int_{B_{r_{i}}\left(x_{i}\right)}|\nabla u|^{s} \mathrm{~d} x\right)^{w / s} \mathrm{~d} t\right)^{2 / w}\left(\int_{t_{i}-r_{i}^{2}}^{t_{i}} \mathrm{~d} t\right)^{1-\frac{2}{w}} .
\end{aligned}
$$

Hence

$$
\varepsilon r_{i} \leq c\left(r_{i}\right)^{3\left(1-\frac{2}{s}\right)+2\left(1-\frac{2}{w}\right)}\left(\int_{t_{i}-r_{i}^{2}}^{t_{i}}\left(\int_{B_{r_{i}}\left(x_{i}\right)}|\nabla u|^{s} \mathrm{~d} x\right)^{w / s} \mathrm{~d} t\right)^{2 / w}
$$

and finally

$$
c^{-w / 2} \varepsilon^{w / 2}\left(r_{i}\right)^{\beta} \leq \int_{t_{i}-r_{i}^{2}}^{t_{i}}\left(\int_{B_{r_{i}}\left(x_{i}\right)}|\nabla u|^{s} \mathrm{~d} x\right)^{w / s} \mathrm{~d} t,
$$

where $\beta=w\left(\frac{3}{s}+\frac{2}{w}-2\right)$. 
Now for $i=1,2,3, \ldots$, let

$$
\varphi_{i}(t)=\int_{B_{r_{i}}\left(x_{i}\right)}|\nabla u(x, t)|^{s} \mathrm{~d} x \quad \text { if } \quad t_{i}-r_{i}^{2} \leq t \leq t_{i},
$$

and $\varphi_{i}(t)=0$ otherwise. Since

$$
\int_{t_{i}-r_{i}^{2}}^{t_{i}}\left(\int_{B_{r_{i}}\left(x_{i}\right)}|\nabla u|^{s} \mathrm{~d} x\right)^{w / s} \mathrm{~d} t=\int_{0}^{T}\left[\varphi_{i}(t)\right]^{w / s} \mathrm{~d} t,
$$

it follows that for $C=c^{w / 2} \varepsilon^{-w / 2}$ we have

$$
\sum_{i=1}^{\infty} r_{i}^{\beta} \leq C \sum_{i=1}^{\infty} \int_{0}^{T}\left[\varphi_{i}(t)\right]^{w / s} \mathrm{~d} t \leq C \int_{0}^{T}\left[\sum_{i=1}^{\infty} \varphi_{i}(t)\right]^{w / s} \mathrm{~d} t .
$$

Since the cylinders are disjoint, for each $0 \leq t \leq T$ we have

$$
\sum_{i=1}^{\infty} \varphi_{i}(t) \leq \int_{\Omega}|\nabla u(x, t)|^{s} \mathrm{~d} x .
$$

Now let $\Pi \mathcal{S}$ be the projection of $\mathcal{S}$ onto $(0, T)$ and let $\mathcal{T}$ be the set of singular times:

$$
\mathcal{T}=\mathbb{R} \backslash \bigcup_{q=1}^{\infty} J_{q}
$$

where $J_{q}$ are intervals of regularity of a weak solution $u$ (the existence of such "epochs of regularity" dates back to [10]). The set $\mathcal{T}$ has box-counting dimension no greater than $1 / 2$ ([14]). Moreover ${ }^{1}, \Pi \mathcal{S} \subseteq \mathcal{T}$. Indeed, if $t_{0} \in J_{q}$ for some $q$, then for sufficiently small $\varepsilon>0$ the weak solution $u$ is uniformly bounded for all $(x, t) \in\left(t_{0}-\varepsilon, t_{0}+\varepsilon\right) \times \Omega$. It follows that if $D$ is the projection of $\mathcal{C}^{\prime}$ onto $(0, T)$ then $D \subseteq O_{\delta}(\mathcal{T})$, where $O_{\delta}(\mathcal{T})$ is $\delta$-neighbourhood of $\mathcal{T}$. In particular, since $d_{B}(\mathcal{T}) \leq 1 / 2$, for any $\theta>1 / 2$, one can cover $O_{\delta}(\mathcal{T})$ and hence $D$ by $c \delta^{-\theta}$ intervals of length $4 \delta$, and hence $\mu(D) \leq c \delta^{-\theta}(4 \delta) \rightarrow 0$ as $\delta \rightarrow 0$.

We have

$$
\sum_{i=1}^{\infty} r_{i}^{\beta} \leq C \int_{0}^{T}\left[\sum_{i=1}^{\infty} \varphi_{i}(t)\right]^{w / s} \mathrm{~d} t \leq C \int_{D}\left(\int_{\Omega}|\nabla u|^{s} \mathrm{~d} x\right)^{w / s} \mathrm{~d} t
$$

where the right-hand side tends to zero as $\delta \rightarrow 0$ (since the integrand is in $L^{1}(0, T)$ ).

To summarise, given any $\delta>0$ we have found a covering of $\mathcal{S}$ by sets $Q_{5 r_{i}}\left(x_{i}, t_{i}\right)$ such that $5 r_{i}<\delta$ and

$$
\sum_{i=1}^{\infty}\left(5 r_{i}\right)^{\beta} \leq 5^{\beta} C \int_{D}\left(\int_{\Omega}|\nabla u|^{s} \mathrm{~d} x\right)^{w / s} \mathrm{~d} t,
$$

where the right hand side of the above inequality tends to zero as $\delta$ tends to zero. It follows that $\mathcal{P}^{\beta}(\mathcal{S})=0$, and hence that $d_{H}(\mathcal{S}) \leq d_{P H}(\mathcal{S}) \leq \beta$.

\footnotetext{
${ }^{1}$ One can show equality in the case of periodic boundary conditions, see [12]. The proof there - which relies on a compactness argument - does not obviously generalise to $\mathbb{R}^{3}$ or a bounded domain.
} 
Note that if $\beta=0$ then the set of singular points is empty. Indeed, if $(x, t)$ is a singular point, then taking into account that

$$
\limsup _{r \rightarrow 0} \frac{1}{r} \int_{Q_{r}(x, t)}|\nabla u|^{2} \mathrm{~d} x \mathrm{~d} t>\varepsilon
$$

and reasoning as above we can show that there exists a sequence $r_{n} \rightarrow 0$ such that

$$
\varepsilon^{w / 2} \leq \int_{t-r_{n}^{2}}^{t}\left(\int_{B_{r_{n}}(x)}|\nabla u|^{s} \mathrm{~d} x\right)^{w / s} \mathrm{~d} t \leq \int_{t-r_{n}^{2}}^{t}\left(\int_{\Omega}|\nabla u|^{s} \mathrm{~d} x\right)^{w / s} \mathrm{~d} t ;
$$

but the right-hand side tends to zero as $n$ tends to infinity, which is a contradiction.

\section{Conclusion}

In this paper we presented the upper bounds on the box-counting and Hausdorff dimension of the singular set of a suitable weak solution to the Navier-Stokes equations that has some additional regularity. As the border cases of these bounds we have obtained the well-known conditions for regularity of weak solutions.

Some natural questions arise from these results. It would be interesting to relax the assumption $w>2$ in Theorem 1 and obtain the same bound for any $w \geq 2$; similarly in Theorem 2 one would like to relax the condition $w \geq s$. In order to obtain Theorem 1 in a bounded domain we would require the analogue of Lemma 2 (estimates for the pressure when $\left.u \in L^{w}\left(0, T ; L^{s}(\Omega)\right)\right)$.

An order of magnitude harder is to determine whether any of these partial regularity results can be proved for general weak solutions, and not only suitable weak solutions.

Acknowledgements. Both JCR and WS are supported by the EPSRC grant EP/G007470/1; WS is also supported by the Polish Government grant N N201 547438.

Open Access This article is distributed under the terms of the Creative Commons Attribution Noncommercial License which permits any noncommercial use, distribution, and reproduction in any medium, provided the original author(s) and source are credited.

\section{References}

1. Beirão da Veiga, H.: A new regularity class for the Navier-Stokes equations in $R^{n}$. Chinese Ann. Math. Ser. B 16(4), 407-412 (1995)

2. Caffarelli, L., Kohn, R., Nirenberg, L.: Partial regularity of suitable weak solutions of the Navier-Stokes equations. Commun. Pure Appl. Math. 35, 771-831 (1982)

3. Constantin, P., Foias, C.: Navier-Stokes Equations. Chicago, IL: University of Chicago Press, 1988

4. Escauriaza, L., Seregin, G., Šverák, V.: $L_{3, \infty}$-Solutions to the Navier-Stokes equations and backward uniqueness. Russ. Math. Surv. 58(2), 211-250 (2003)

5. Fabes, E.B., Jones, B.F., Rivière, M.M.: The initial value problem for the Navier-Stokes equations with data in $L^{p}$. Arch. Rat. Mech. Anal. 45, 222-240 (1972)

6. Falconer, K.: Fractal Geometry: Mathematical Foundations and Applications. (2 ${ }^{\text {nd }}$ edition), Chichester: John Wiley \& Sons, 2003

7. Hopf, E.: Über die Anfangswertaufgabe dür die hydrodynamischen Grundgleichungen. Math. Nachr. 4, 213-231 (1951)

8. Kukavica, I.: The fractal dimenson of the singular set for solutions of the Navier-Stokes system. Nonlinearity 22, 2889-2900 (2009)

9. Ladyzhenskaya, O., Seregin, G.: On partial regularity of suitable weak solutions to the three-dimensional Navier-Stokes equations. J. Math. Fluid Mech. 1, 356-387 (1999) 
10. Leray, J.: Essai sur le mouvement d'un fluide visqueux emplissant l'espace. Acta Math. 63, 193-248 (1934)

11. Lin, F.: A New Proof of the Caffarelli-Kohn-Nirenberg Theorem. Comm. Pure Appl. Math. 51, 241-257 (1998)

12. Robinson, J.C.: Regularity and singularity in the three-dimensional Navier Stokes equations. Bole. Soc. Española de Mate. Apli. 35, 43-71 (2006)

13. Robinson, J.C.: Dimensions, Embeddings, and Attractors. Cambridge Tracts in Mathematics, Vol. 186. Cambridge: Cambridge University Press, 2011

14. Robinson, J.C., Sadowski, W.: Decay of weak solutions and the singular set of the three-dimensional Navier-Stokes equations. Nonlinearity 20, 1185-1191 (2007)

15. Robinson, J.C., Sadowski, W.: Almost-everywhere uniqueness of Lagrangian trajectories for suitable weak solutions of the three-dimensional Navier-Stokes equations. Nonlinearity 22, 2093-2099 (2009)

16. Scheffer, V.: Turbulence and Hausdorff dimension. In: Turbulence and Navier-Stokes equations, Orsay 1975, Springer LNM 565, Berlin: Springer-Verlag, 1976, pp. 174-183

17. Serrin, J.: On the interior regulariy of weak solutions of the Navier-Stokes equations. Arch. Rat. Mech. Anal. 9, 187-191 (1962)

18. Struwe, M.: On partial regularity results for the Navier-Stokes equations. Comm. Pure Appl. Math 41(4), 437-458 (1988)

19. Wolf, J.: A new criterion for partial regularity of suitable weak solutions to the Navier-Stokes equations. In: Rannacher, R., Sequeira, A. (eds.) Advances in Mathematical Fluid Mechanics, Springer-Verlag, New York, USA (2010)

Communicated by P. Constantin 